\title{
STUDY ON SLEEP STRUCTURE AND INFLUENCING FACTORS OF EPILEPSY CHILDREN WITH SLEEP DISORDER
}

\author{
Z. Ning, J. Zhang, J. Zhang, B. Chen, L. Yang \\ Neurology, Hunan Children's Hospital, Changsha, China
}

Objective: This study was conducted to find out the sleep structure of children hospitalized with epilepsy, and to explore the influencing factors of epilepsy with sleep disorders, in order to provide a reference of interventions to reduce the incidence of sleep disorders in epileptic children, to promote physical and mental healthy development of epileptic children.

Methods: 111 patients were diagnosed epilepsy with sleep disorders in Hunan children"s hospital from December 2006 to June 2010, as a case group; while select 89 epileptic children not accompanied sleep disorders as the control group at the same time.

\section{Results:}

(1) The general information description: epilepsy with sleep disorders group (case group) 111 cases, sex ratio was 1.31:1, epileptic children not accompanied sleep disorders (control group) 89cases, sex ratio was 0.31:1.

(2) Sleep structure: compared with the control group, case group had significant sleep structure disorder.

(3) Those epileptic children who have sleep disturbance, hyperactivity or frequent awakening, difficulty falling asleep, and whose family sleep environment is not quiet were apt to lead sleep disorders.

Conclusion: Epilepsy children with sleep disorder have significant changes in sleep structure, the occurrence of sleep disorders affected by many factors. Health education of sleeping should be enhanced. Early monitoring and guidance of sleep can improve the quality of life of epileptic children. 